\title{
Antibiotic prophylaxis for infections in patients with acute stroke: a systematic review and meta-analysis of randomized controlled trials
}

\author{
Yan-Guo Xi' ${ }^{1, *}$, Xu Tian ${ }^{2, *}$, Wei-Qing Chen ${ }^{2, *}$, Sai Zhang ${ }^{3, *}$, Shan Zhang ${ }^{1}$, Wei-Dan Ren ${ }^{1}$, \\ Qi-Jun Pang ${ }^{1}$, Guo-Tao Yang ${ }^{1}$ and Zhi-Ming Yang ${ }^{1}$ \\ ${ }^{1}$ Department of Neurosurgery, Cang Zhou Central Hospital, Hebei 061001, China \\ ${ }^{2}$ Department of Gastroenterology, Chongqing Cancer Institute and Hospital and Cancer Center, Chongqing 400030, China \\ ${ }^{3}$ Department of Neurosurgery, Logistic University Affiliated Hospital, Logistic University of Chinese People's Armed Police \\ Force, Tianjin 300162, China \\ *These authors contributed equally to this work \\ Correspondence to: Yan-Guo Xi, email: neuroxi@163.com \\ Xu Tian, email: yxtx880919@hotmail.com \\ Keywords: acute stroke, antibiotic prophylaxis, systematic review, meta-analysis
}

Received: March 22, 2017 Accepted: June 17, $2017 \quad$ Published: July 06, 2017

Copyright: Xi et al. This is an open-access article distributed under the terms of the Creative Commons Attribution License 3.0 (CC BY 3.0), which permits unrestricted use, distribution, and reproduction in any medium, provided the original author and source are credited.

\section{ABSTRACT}

Objective: Infections are frequent after stroke and lead to increased mortality and neurological disability. Antibiotic prophylaxis has potential of decreasing the risk of infections and mortality and improving poor functional outcome. Several studies evaluated antibiotic prophylaxis for infections in acute stroke patients have generated conflicting results. The systematic review of randomized clinical trials (RCTs) aimed at comprehensively assessing the evidence of antibiotic prophylaxis for the treatment of acute stroke patients.

Materials and Methods: PubMed, EMBASE, the Cochrane library and the reference lists of eligible articles were searched to identify all potential studies. We included the studies that investigated the efficacy and safety of antibiotic prophylaxis for the treatment of acute stroke patients. The primary outcome included mortality and infection rate. The secondary outcomes included poor functional outcome and adverse events.

Results: Seven trials randomizing 4,261 patients were included. Pooled analyses showed that antibiotic prophylaxis did not improve the mortality (risk ratio (RR) $=1.03,95 \%$ confidence interval (CI) 0.84 to $1.26, p=0.78, I^{2}=25 \%$ ) and poor functional outcome ( $R R=0.93,95 \%$ CI 0.80 to $1.08, p=0.32, I^{2}=80 \%$ ), but reduced the incidence of infection ( $R R=0.67,95 \% C I 0.53$ to $0.84, p=0.0007, I^{2}=49 \%$ ). No major side effects were reported. Sensitivity analyses confirmed the results of infection rate and poor functional outcome.

Conclusions: Antibiotic prophylaxis can be used to treat the infectious events of acute stroke patients although it has no potential of decreased mortality and improved functional outcome.

\section{INTRODUCTION}

Stroke is a major contributor to cardiocerebral vascular diseases-related disability and death worldwide, especially in low- and middle-income countries $[1,2]$. Stroke not only impairs many vital neurological functions, but causes severe complications such as infections [3]. Previous definitions used for diagnosing infection were substantially different, and hence it was defined predominately based on the modified Centers for Disease Control and Prevention criteria [4]. About 5-65\% of acute stroke patients are at risk of infection [5]. A meta-analysis 
including 137,817 patients reported an overall infection rate of $30 \%$, as for pneumonia and urinary tract infection (UTI), the corresponding rate was $10 \%$ [5]. In intensive care units (ICU), the infection rate was up to $45 \%$ [5]. Of patients with post-stroke infection, $48 \%$ died compared to $18 \%$ patients without infection [5]. Several studies well demonstrated that post-stroke infection was associated with increased mortality and poor functional outcome [6, 7]. Thus, it is extremely important to effectively and successfully manage the infection following the stroke.

Published evidences demonstrated that antibiotic prophylaxis therapy may decrease the risk of infection [8] and mortality [9] and improve the functional outcome. Previous experimental studies $[10,11]$ demonstrated that antibiotic prophylaxis reduced post-stroke infection and also improved other clinical outcomes. Moreover, several animal studies [12-14] also suggested that some antibiotics have a potential neuroprotective effect. Of these antibiotics, Minocycline and ceftriaxone have been shown to improve neurological performance and survival through inducing glutamate transporter expression, stimulating neurotrophins or suppressing the release of inflammatory cytokines. However, the relevant clinical trials of investigated the efficacy of antibiotic prophylaxis in treating acute stroke patients generated conflicting findings [9, 15-20]. The PANTHERIS trial [16], a randomized, double-blind, and placebo-controlled trial, recruited 79 patients who suffered acute ischemic stroke with a National Institute of Health Stroke Scale (NIHSS) score $>11$. Administration of moxifloxacin was intravenously initiated within 36 hours after stroke onset, with $400 \mathrm{mg}$ daily for 5 days. Moxifloxacin reduced the infection rate from $32.5 \%$ to $15.4 \%$ but did not significantly improve neurological outcome and survival. Lampl [18] performed another randomized controlled trial (RCT) in Israel, in which they recruited 141 patients with NHISS $>5$ under 5-day taking Minocycline treatment at a dosage of $200 \mathrm{mg}$. The result illustrated that, compared with placebo, patients assigned to minocycline obtained superior outcome on the followed 7th day and 30th day. Chamorro [15] also designed a RCT in Spain, which recruited 136 patients with a NIHSS $\geq 5$ for intravenous levofloxacin treatment of 3-day duration. Controversial results were reported that prophylactic administration of levofloxacin was not better than optimal care. It is must be noted that the current guidelines did not recommend antibiotic prophylaxis to treat acute stroke patients [21]. More importantly, a recent large-scale study suggested [17] that antibiotic prophylaxis did not reduce incidence of post-stroke pneumonia in stroke patients with dysphagia. Two prospective and multicenter trials also concluded that antibiotic prophylaxis did not improve functional outcome at 3 months in acute stroke patients $[17,19]$. As a result, it remains controversial whether antibiotic prophylaxis has the potential of reducing rate of infections, mortality and disability in stroke patients. And thus, we designed this systematic review to comprehensively assess the effects of antibiotic prophylaxis on post- stroke infections and functional outcome in acute stroke patients.

\section{RESULTS}

\section{Description of the studies}

We initially captured 471 records and 13 articles were included to evaluate the eligibility based on fulltext. Six studies were excluded because of ineligible participant, ineligible intervention and lack of eligible outcomes of interest and 7 RCTs were included into qualitative synthesis eventually. The Figure 1 showed the process of searching and screening of studies.

Characteristics of included studies are documented in Table 1. These studies [9, 15-20] enrolling 4,261 stroke patients (2131 in antibiotics groups versus 2130 in control groups). The sample size of individual study varied from 60 to 2538 . In the control groups, 228 patients $[15,16,18,20]$ were randomized to receive placebo and 1902 patients [9, 17, 19] have no additional treatment. The basic median stroke severity scores were based on the National Institute of Health Stroke Scale (NIHSS) in six studies [9, 15-19]. These scores ranged from 7.6 to 15 in control groups versus 7.5 to 17 in antibiotics groups. The Canadian Neurological Scale (CNS) was used to assess stroke severity in one study [20] (median score of 4.1 in control groups versus 4.5 in antibiotic groups). Three studies [15, 17, 19] enrolled ischemic and hemorrhagic stroke patients, whereas the remaining four studies $[9,16,18,20]$ only recruited ischemic stroke patients. Intervention regimes differed in these seven studies: fluoroquinolones (levofloxacin [15], moxifloxacin [16]) were used in two studies, ceftriaxone [19] in one study, penicillin [20] in one study, minocycline [18] in one study, amoxicillin or co-amoxiclav, together with clarithromycin [17] in one study and a combination of $\beta$-lactam antibiotic with $\beta$-lactamase inhibitor [9] in one study. Route of administration included intravenous [9, 15-17, 19], intramuscular [20] and oral [18]. Duration of preventive antibiotic therapy ranged from 3 to 7 days and was not noted in one study [20].

\section{Quality assessment}

The assessment of the risk of bias for individual studies is delineated in Figure 2. All studies generated random sequence appropriately. Allocation concealment was not referred in one study [20]. One study used the 8th number of the participant's identity card (ID) for randomization, which could be concluded that treatment allocation was not concealed because physicians could know patient's ID numbers and get a certain treatment. Due to open-label design, 4 [9, 17-19] out of 7 studies have performance bias. Of these, 3 studies [17-19] 
described masked endpoint assessment. One study [9] described blinded assessment of infections but did not describe blinded assessment of secondary outcomes, such as NIHSS and mRS. Blinding was not specified in one study [20]. Two studies [18, 20] did not report the losses of follow-up. One study [16] described the loss of follow-up, but did not mention the further details. Only per-protocol analysis was executed in one study [20]. The whole quality of all included studies was judged as moderate.

\section{Mortality}

Results of all 7 studies were available for the analysis of mortality at the end of follow-up. Meta-analysis showed that antibiotic prophylaxis had no effect on overall mortality $(\mathrm{RR}=1.03,95 \%$ CI 0.84 to $1.26, p=0.78)$ (See Figure 3). There was no substantial heterogeneity for mortality $\left(I^{2}=25 \%, p=0.24\right)$ (See Figure 3$)$.

\section{Infection rate}

The definitions for the diagnosis of infection differed substantially across trials (See Table 2). Thus, we used infection rate defined by the investigators. One study [18] did not report infection rate. The pooled effect size in the remaining 6 showed an association between antibiotic prophylaxis and reduced incidence of infection $(\mathrm{RR}=$ $0.67,95 \%$ CI 0.53 to $0.84, p=0.00$ ) (See Figure 4). No significant heterogeneity was found among the identified comparisons $\left(I^{2}=49 \%, P=0.08\right)$ (See Figure 4$)$.

\section{Poor functional outcome}

One trial [20] did not report the number of patients with poor outcome at the endpoint assessment. So the data on poor functional outcome was available in 6 trials. The pooled RR of poor functional outcome was $0.93(95 \%$
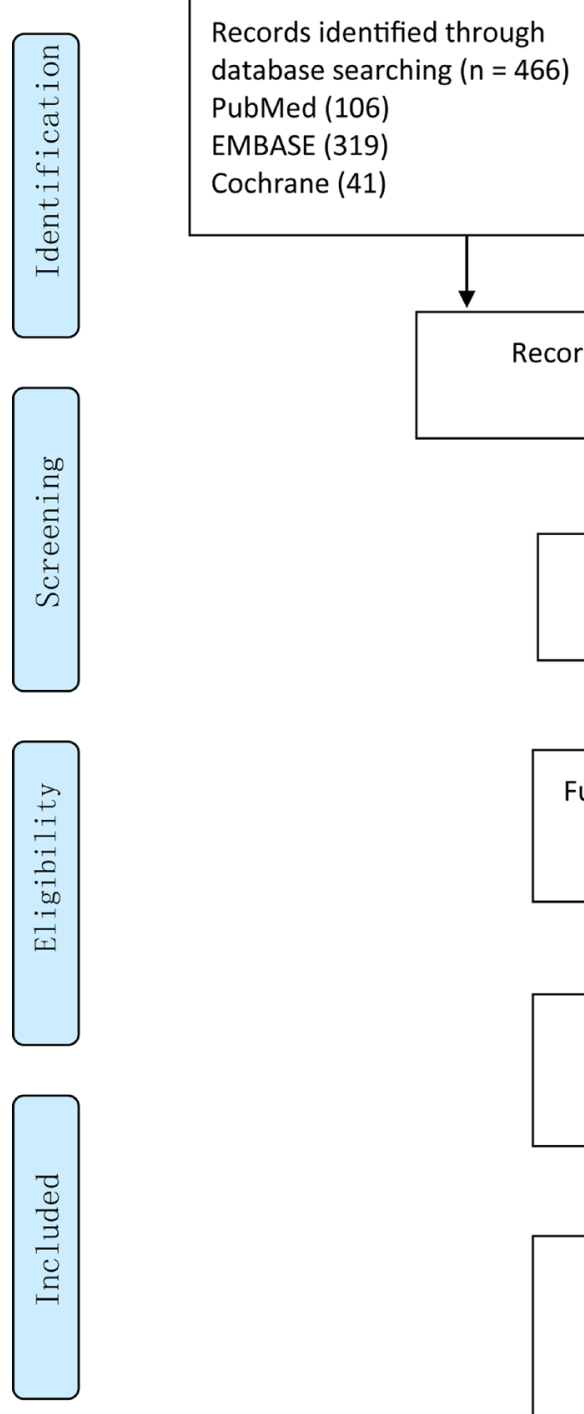

Records after duplicates removed $(n=408)$

Additional records identified through other sources $(n=5)$

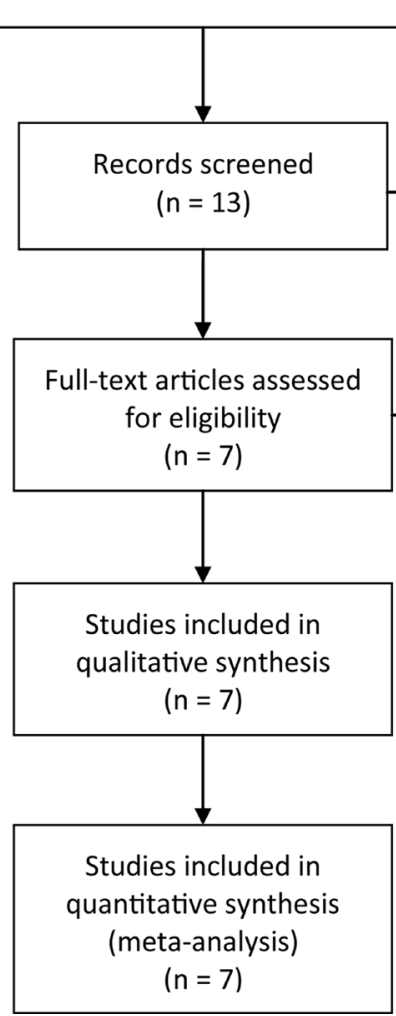

Records excluded $(n=395)$

Animal research $(n=3)$

Review articles $(n=16)$

Unrelated to the topic $(n=355)$

Letters/editorials/comment $(n=2)$

Protocol $(n=9)$

Overlap studies reported at different years $(n=9)$ No-RCT (4)

Full-text articles excluded, with reasons $(n=6)$ Ineligible participant $(n=1)$ Ineligible intervention $(n=2)$ Not outcome of interest $(n=3)$ Conference abstract $(n=1)$

Figure 1: Flow chart of retrieval and selection of literatures. $\mathrm{RCT}$ = randomized controlled trials. 
Table 1: Characteristics of the included studies

\begin{tabular}{|c|c|c|c|c|c|c|c|}
\hline Author (year) & Study design & Inclusion criteria & Exclusion Criteria & Sample & $\begin{array}{l}\text { Treatment arms } \\
\text { (No) }\end{array}$ & Intervention & $\begin{array}{c}\text { Outcomes of } \\
\text { interest }\end{array}$ \\
\hline $\begin{array}{l}\text { Chamorro } \\
2005^{15}\end{array}$ & RCT double-blind & $\begin{array}{c}\text { Ischemic stroke } \\
<12 \text { hours; Age } \geq 18 \\
\text { years, (NIHSS) } \geq 5\end{array}$ & $\begin{array}{c}\text { Infection }<3 \mathrm{mo} ; \mathrm{T}>37.7^{\circ} \mathrm{C} \text {; allergy } \\
\text { to fluoroquinolones; epilepsy; seizures; } \\
\text { serum creatinine }>2.5 \mathrm{mg} / \mathrm{dL} \text {, antibiotics } \\
\text { use; immunosuppressants therapy }<3 \mathrm{mo}\end{array}$ & 136 & $\begin{array}{l}\text { Antibiotics (67) vs } \\
\text { placebo (69) }\end{array}$ & $\begin{array}{l}\text { Levofloxacin } 500 \mathrm{mg} / \mathrm{d} \\
\text { IV for } 3 \mathrm{~d} \text {, started within } \\
24 \mathrm{~h} \text { of stroke onset }\end{array}$ & $\begin{array}{l}\text { 7-day infection, } \\
\text { 3-month neurological } \\
\text { outcome and } \\
\text { mortality }\end{array}$ \\
\hline Harms $2008^{16}$ & RCT double-blind & $\begin{array}{c}\text { Ischemic stroke }(9-36 \\
\text { hours); Age } \geq 18 \text { years, } \\
\text { NIHSS }>11\end{array}$ & $\begin{array}{c}\text { infections; Hemorrhagic stroke; } \\
\text { antibiotics therapy }<24 \mathrm{~h} ; \\
\text { contraindications against moxifloxacin; } \\
\text { immunosuppressant treatment }\end{array}$ & 79 & $\begin{array}{l}\text { Antibiotics (39) vs } \\
\text { placebo (40) }\end{array}$ & $\begin{array}{l}\text { Moxifloxacin } 400 \mathrm{mg} / \mathrm{d} \\
\text { IV for } 5 \mathrm{~d} \text {, started within } \\
36 \mathrm{~h} \text { of stroke onset }\end{array}$ & $\begin{array}{l}\text { 11-day infection, } \\
\text { 6-month neurological } \\
\text { outcome and } \\
\text { mortality }\end{array}$ \\
\hline Kalra $2015^{17}$ & RCT open-label & $\begin{array}{c}\text { Stroke }<48 \text { hours; Age } \\
\geq 18 \text { years, with dysphagia }\end{array}$ & $\begin{array}{l}\text { Infections; allergic to antibiotics; } \\
\text { preexisting dysphagia; pyrexia; } \\
\text { pregnancy; imminent death }\end{array}$ & 1217 & $\begin{array}{l}\text { Antibiotics (615) } \\
\text { vs control (602) }\end{array}$ & $\begin{array}{l}\text { Amoxicillin or co- } \\
\text { amoxiclav, together with } \\
\text { clarithromycin IV for } 7 \\
\text { d, started within } 48 \text { h of } \\
\text { stroke onset }\end{array}$ & $\begin{array}{l}\text { 14-day infection, } \\
\text { 3-month neurological } \\
\text { outcome and } \\
\text { mortality }\end{array}$ \\
\hline De Falco $1998^{20}$ & RCT open-label & $\begin{array}{l}\text { Ischemic stroke } \\
<12 \text { hours; All ages }\end{array}$ & NA & 80 & $\begin{array}{l}\text { Antibiotics (38) vs } \\
\quad \text { control (42) }\end{array}$ & $\begin{array}{c}\text { Penicillin } \\
\text { intramuscularly, started } \\
\text { within } 12 \mathrm{~h} \text { of stroke } \\
\text { onset }\end{array}$ & $\begin{array}{l}\text { In-hospital infection } \\
\text { and mortality }\end{array}$ \\
\hline Lampl $2007^{18}$ & RCT open-label & $\begin{array}{l}\text { Ischemic stroke }(6-24 \\
\text { hours); Age } \geq 18 \text { years, } \\
\text { NIHSS }>5\end{array}$ & $\begin{array}{l}\text { Hemorrhagic stroke; other disease; pre- } \\
\text { existing neurologicdisability; tetracycline } \\
\text { allergic; renal failure; pre- existing } \\
\text { infectious disease; swallowing difficulties }\end{array}$ & 151 & $\begin{array}{l}\text { Antibiotics (74) vs } \\
\text { placebo (77) }\end{array}$ & $\begin{array}{c}\text { Minocycline } 200 \mathrm{mg} / \mathrm{d} \\
\text { orally for } 5 \mathrm{~d} \text {, started } \\
\text { within } 6-24 \mathrm{~h} \text { of stroke } \\
\text { onset }\end{array}$ & $\begin{array}{l}\text { 3-month neurological } \\
\text { outcome and } \\
\text { mortality }\end{array}$ \\
\hline Schwarz $2008^{9}$ & RCT open-label & $\begin{array}{c}\text { Ischemic stroke }<24 \\
\text { hours; Age } \geq 18 \text { years, } \\
\text { mRS }>3\end{array}$ & $\begin{array}{l}\text { Infections; hemorrhagic stroke; renal } \\
\text { insufficiency; penicillin or sulbactam } \\
\text { allergic; immunosuppressant treatment; } \\
\text { pregnancy }\end{array}$ & 60 & $\begin{array}{l}\text { Antibiotics (30) vs } \\
\text { control (30) }\end{array}$ & $\begin{array}{l}\text { Mezlocillin } 6 \mathrm{~g} / \mathrm{d} \text { plus } \\
\text { sulbactam } 1 \mathrm{~g} / \mathrm{d} \text { IVfor } \\
4 \mathrm{~d}, \text { started within } 24 \mathrm{~h} \\
\text { of stroke onset }\end{array}$ & $\begin{array}{l}\text { 10-day infection, } \\
\text { 3-month neurological } \\
\text { outcome and } \\
\text { mortality }\end{array}$ \\
\hline $\begin{array}{l}\text { Westendorp } \\
2015^{19}\end{array}$ & RCT open-label & $\begin{array}{c}\text { Stroke }<24 \text { hours; Age } \geq \\
18 \text { years, NIHSS } \geq 1\end{array}$ & $\begin{array}{l}\text { Infections; antibiotics therapy }<24 \mathrm{~h} \text {; } \\
\text { pregnancy; penicillin or cephalosporins } \\
\text { allergic; subarachnoid hemorrhage; }\end{array}$ & 2538 & $\begin{array}{c}\text { Antibiotics (1268) } \\
\text { vs control (1270) }\end{array}$ & $\begin{array}{l}\text { Ceftriaxone } 2 \mathrm{~g} / \mathrm{d} \text { IV for } \\
4 \mathrm{~d} \text {, started within } 24 \mathrm{~h} \\
\text { of stroke onset }\end{array}$ & $\begin{array}{l}\text { In-hospital infection, } \\
\text { 3-month neurological } \\
\text { outcome and } \\
\text { mortality }\end{array}$ \\
\hline
\end{tabular}

RCTs = randomized controlled trials; NIHSS = National Institute of Health Stroke Scale; $\mathrm{mRS}=$ modified Rankin Score; $v \mathrm{~s}=$ versus; $\mathrm{mo}=\mathrm{month} ; \mathrm{t}=\mathrm{temperature} ; \mathrm{NA}=$ not available.

CI 0.80 to $1.08, p=0.32$ ) (See Figure 5). We identified the evidence of statistical heterogeneity $\left(I^{2}=80 \%, p<0.00\right)$ (See Figure 5).

\section{Adverse events}

Medication-related AEs were reported in 4 articles $[9,16,17,19]$. In one study [16], AEs related to antibiotic were not described; however, one patient assigned in antibiotics prophylaxis group was reported to suffer from post-stroke pneumonia which was caused by methicillinresistant Staphylococcus aureus (MRSA) ; however, the MRSA has been colonized before the patient enrolled into the present study. One study [9] reported that each participated patients experienced exanthema and elevated liver enzymes. The incidence of Clostridium difficile toxin (CDT)-positive diarrhea (2 of 615 versus 4 of 602) and MRSA colonization (11 of 615 versus 14 of 602) were low and equal between antibiotic prophylaxis and control groups respectively in one study [17]. One study [19] reported that seven and two patients who were assigned into antibiotics prophylaxis group experienced allergic reaction and $C$. difficile infection respectively.

\section{Subgroup analysis}

We performed subgroup analysis according to the study design (double-blinded, open-label and unclear). Two trials $[15,16]$ used a double-blinded design, four trials [9, 17-19] used an open-label design and one trial [20] did not mention blind method. In the subgroup of using double-blinded design, there was no improvement in poor functional outcome ( $\mathrm{RR}=1.02,95 \% \mathrm{CI} 0.78$ to 1.35 , $p=0.86$ ) with the insignificant statistical heterogeneity $\left(I^{2}=17 \%, p=0.27\right)$ (See Figure 5). Infection rate was not significantly reduced in patients receiving antibiotic prophylaxis ( $\mathrm{RR}=0.67,95 \% \mathrm{CI} 0.37$ to $1.21, p=0.19$ ) with the insignificant statistical heterogeneity $\left(I^{2}=11 \%\right.$, $p=0.29$ ) (See Figure 4). In the subgroup of using openlabel design, there was also no improvement in poor functional outcome $(\mathrm{RR}=0.89,95 \%$ CI 0.74 to 1.08 , $p=0.24)$ with the significant statistical heterogeneity $\left(I^{2}=88 \%, p<0.00\right)$ (See Figure 5$)$. The pooled RR of infection rate showed there was association between the antibiotic prophylaxis and the reduced infection rate $(\mathrm{RR}=$ $0.67,95 \%$ CI 0.51 to $0.88, p=0.004)$ with the significant statistical heterogeneity $\left(I^{2}=65 \%, p=0.03\right)$ (See Figure 4).

\section{Sensitivity analysis}

One study [17] used cluster-randomized design which could introduce dependence (or clustering) between individual units sampled. The exclusion of this study did not change the pooled RR of poor functional outcome $(\mathrm{RR}=0.87,95 \% \mathrm{CI} 0.69-1.10, p=0.27)$ nor did it reduce heterogeneity $\left(I^{2}=86 \%\right)$ (See Figure 6) substantially. Moreover, the sensitivity analysis was conducted by excluding the same study and a robust pooled result in 
A Risk of Bias Graph

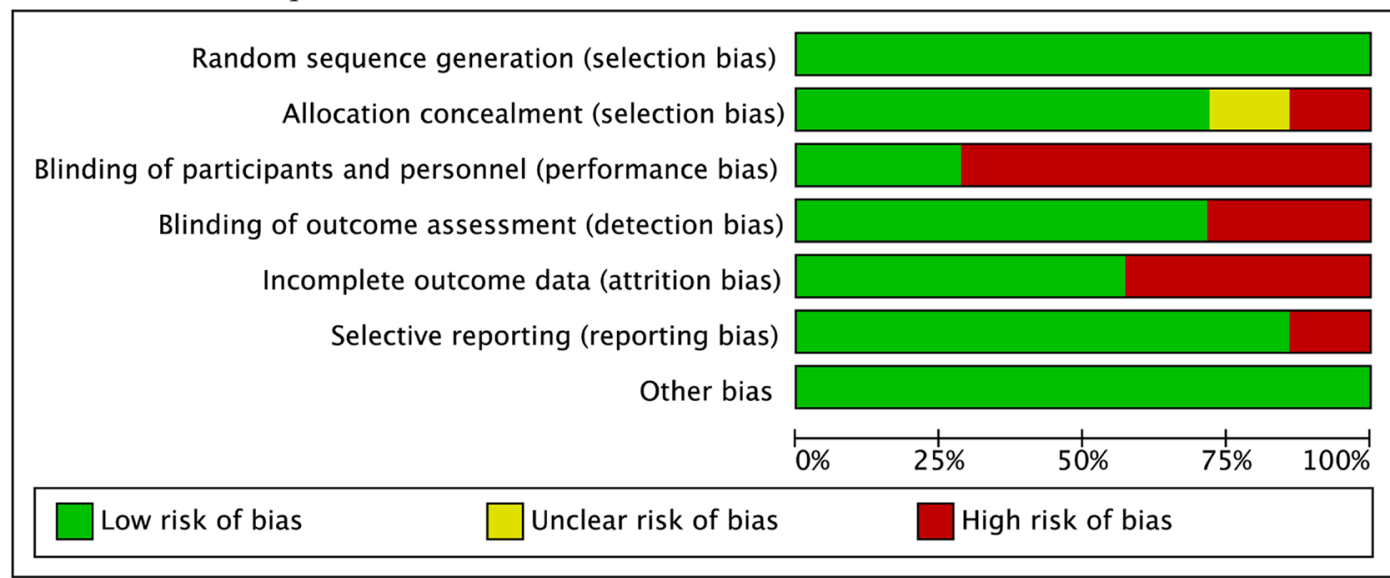

B Risk of Bias Summary

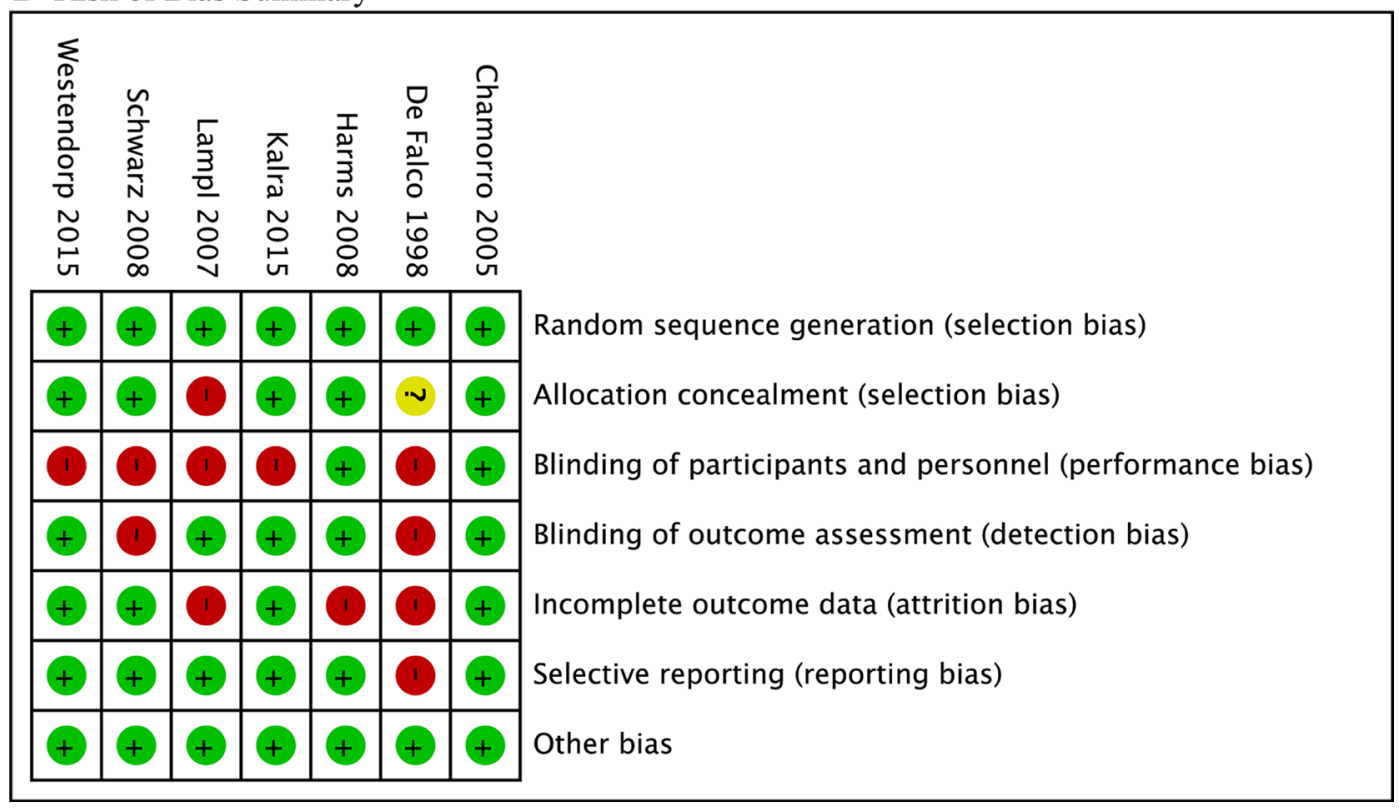

Figure 2: Risk of bias. (A). Risk of bias graph, (B) Risk of bias summary. Green, yellow, and red color indicated low, unclear, and high risk of bias respectively.

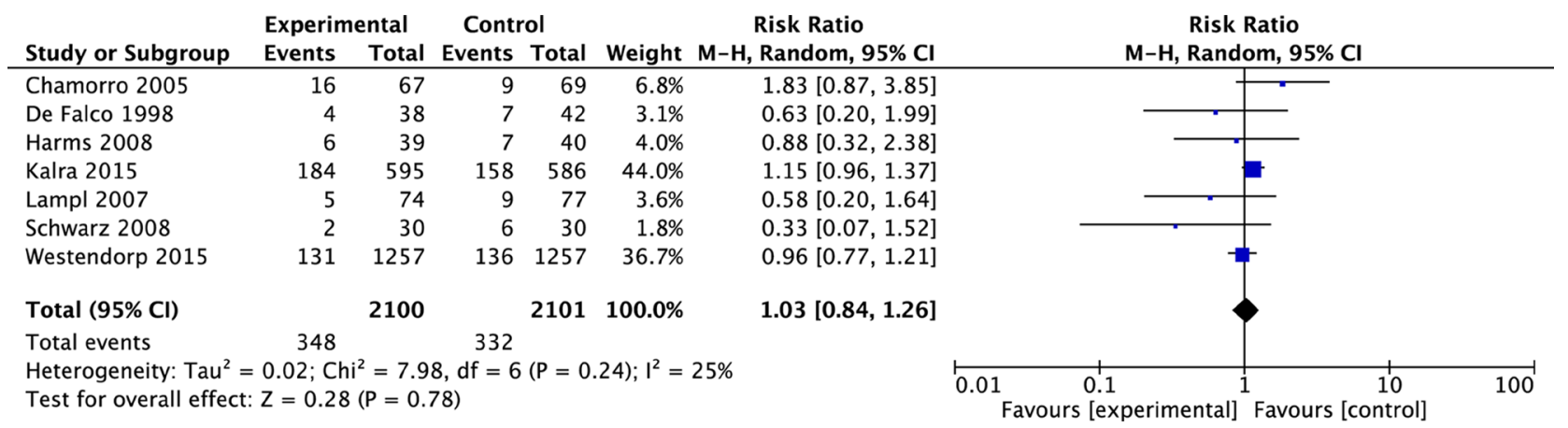

Figure 3: Meta-analysis on mortality. The summary effect estimate (risk ratio, RR) for individual randomized controlled trials (RCTs) are indicated by green rectangles (the size of the rectangle is proportional to the study weight), with the black horizontal lines representing 95 per cent c.i. The overall summary effect estimate and 95 per cent c.i. are indicated by the black diamond below. 
Table 2: Definitions used for infection

Source

Definition

Temperature $>37.5^{\circ} \mathrm{C}$ in two determinations; or $>37.8$ in a single determination in patients with

Chamorro $2005^{15}$ suggestive symptoms; white blood cell count $>11,000 / \mathrm{mL}$ or $<4000 / \mathrm{mL}$; pulmonary infiltrate on chest $\mathrm{x}$-rays, or cultures positive for a pathogen. Early infection: within 7 days, late: 8 to 90 days.

Pneumonia, > 1 of: abnormal respiratory examination, or pulmonary infiltrates in chest x-rays, productive

Harms $2008^{16}$ cough with purulent sputum, microbiological cultures from lower respiratory tract or blood cultures, leukocytosis and elevation of CRP. UTI: $>1$ of the following: fever (temperature $>38.0^{\circ} \mathrm{C}$ ), urine sample positive for nitrite, leucocyturia, and significant bacteriuria.

Kalra $2015^{17} \quad$ Criteria for pneumonia from the Centres for Disease Control and Prevention

De Falco $1998^{20}$

Infectious complications: bronchopulmonary, urinary or hyperthermia of unspecified origin. No definitions specified.

Lampl $2007^{18} \quad$ Not evaluated.

Pneumonia: new infiltrate on chest $x$-ray compatible with the diagnosis of infection plus at least one of the following: fever (temperature $>38^{\circ} \mathrm{C}$ ), leukocytosis $>12,000 / \mu \mathrm{L}$ or leukopenia $<3000 / \mu \mathrm{L}$, purulent tracheal secretions Tracheobronchitis: purulent tracheals secretions or sputum plus at least 1 of the following: fever (temperature $>38^{\circ} \mathrm{C}$ ), leukocytosis $>12,000 / \mu \mathrm{L}$ or leukopenia $<3000 / \mu \mathrm{L}$

Schwarz $2008^{9} \quad$ UTI: $>25$ leukocytes $/ \mu \mathrm{L}$ in the urine if not explained by other findings. Bacteremia: bacteria in blood cultures Sepsis: clinical evidence of an infection with at least two of the following: temperatures $>38^{\circ} \mathrm{C}$ or $<35^{\circ} \mathrm{C}$, tachycardia $>90 /$ minute, tachypnoea $>20 /$ minute, leukocytosis $>12,000 / \mu \mathrm{L}$ or leukopenia $<3000 / \mu \mathrm{L}$ Infection of unclear origin or other infections: clinical evidence of an infection of unknown origin or any other systemic infection

Westendorp First, clinical diagnosis according to the treating physician will be recorded. Second, diagnosis of $2015^{19}$ infection the modified criteria of the United States Centres for Disease Control and Prevention

Abbreviations: CRP, C-reactive protein; UTI, urinary tract infection.

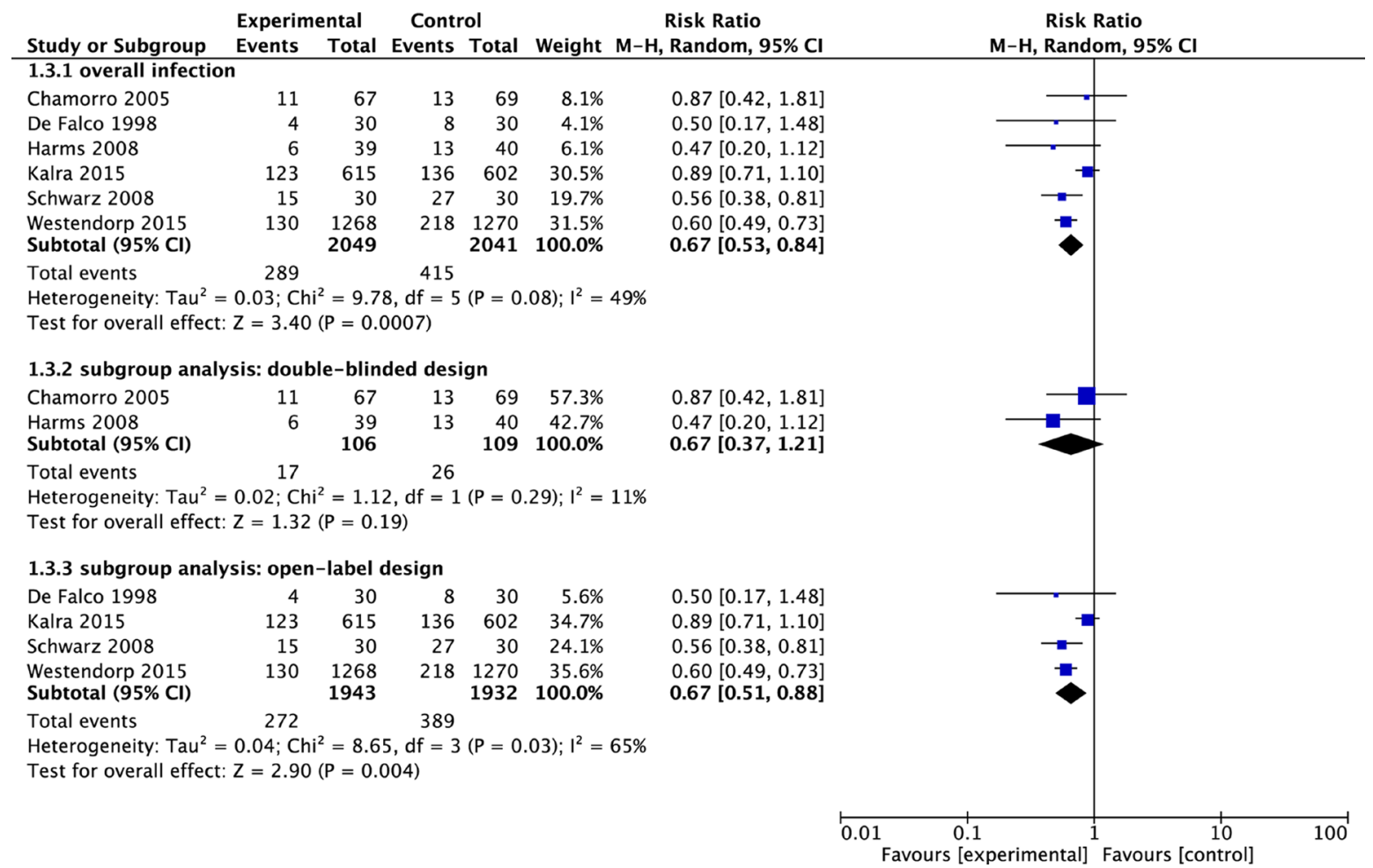

Figure 4: Meta-analysis on infection rate. The summary effect estimate (risk ratio, RR) for individual randomized controlled trials (RCTs) are indicated by green rectangles (the size of the rectangle is proportional to the study weight), with the black horizontal lines representing 95 per cent c.i. The overall summary effect estimate and 95 per cent c.i. are indicated by the black diamond below. 
infections was showed $(\mathrm{RR}=0.59,95 \%$ CI $0.50-0.70$, $p<0.00001$ ) (See Figure 6).

\section{DISCUSSION}

Our present meta-analysis demonstrated that the administration of antibiotic prophylaxis did reduce poststroke infection rate from $14 \%$ to $20 \%$. Prophylactic antibiotic did not affect the mortality and poor functional outcome in patients with acute stroke. Sensitivity analyses showed that infection rate and poor functional outcome were stable. We have found no major AEs of prophylactic antibiotic treatment.

A previous Cochrane review enrolled 5 RCTs showed that antibiotic prophylaxis reduced the infection rate without major AEs [8]. However, the conclusion of this review generated inconclusive findings due to insufficient accumulated sample size of 506 patients [8]. The conclusion was also outdated because of more new RCTs have been carried out since then. Our study included two new large-scale trials and reevaluated the efficacy and safety of prophylactic antibiotic in acute stroke patients.

Infections are frequent after stroke. Despite general measures to prevent infections after stroke onset, pneumonia and UTI remains a common and severe clinical problem even for patients treated in stroke units [7, 22]. Pneumonia can be classified into early (within the first week) and late pneumonia (after the first week) after stroke [23]. Early pneumonia is endogenous and is caused by normal flora carried in the oropharynx on hospital admission. While, delayed pneumonia is resulted from abnormal flora which was acquired in the oropharynx during hospitalization. Recently, a new consensus was reached on the diagnosis of stroke-associated pneumonia: lower respiratory tract infections within the first 7 days after stroke onset [24]. Patients with stroke are vulnerable to UTI due to increased risk of bladder dysfunction, immunosuppression and increased Foley catheter use $[23,25]$. Infections other than pneumonia and UTI occurred in $13 \%$ of stroke patients and included such as skin and intravenous line infections, sepsis and cholecystitis [26]. Many reasons for infection have been postulated, including older age, male sex, stroke severity, dysphagia, gastrointestinal dysmotility and stroke-induced immunodepressive state $[6,27,28]$, and so on. Strokeinduced immunodeficiency via the hypothalamic axis and the sympathetic nervous system can be detected within a few hours after cerebral ischemia and may persist over a few weeks [29]. The immunosuppression may increase the susceptibility to the infections in patients after stroke [29].

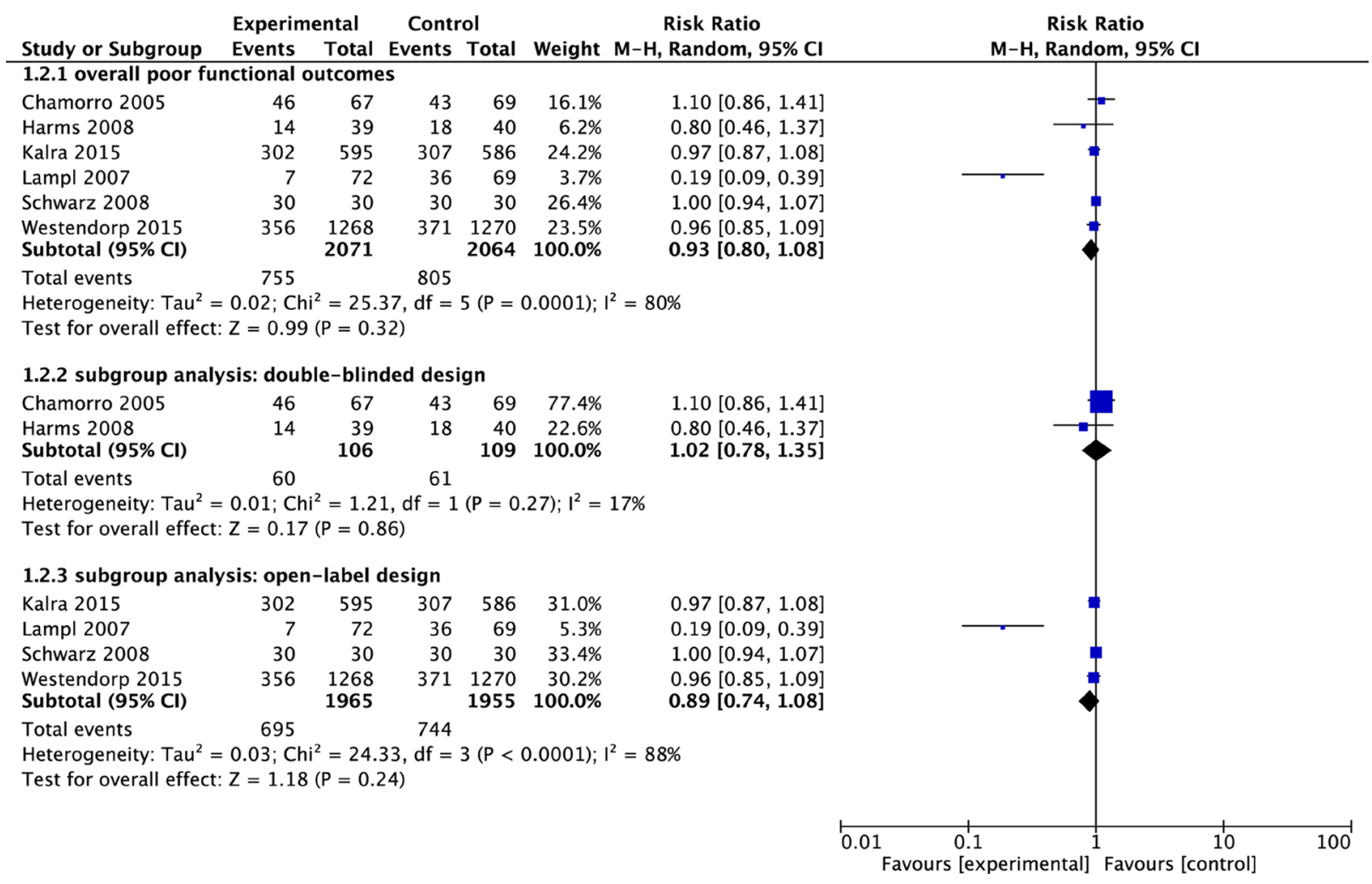

Figure 5: Meta-analysis on poor functional outcomes. The summary effect estimate (risk ratio, RR) for individual randomized controlled trials (RCTs) are indicated by green rectangles (the size of the rectangle is proportional to the study weight), with the black horizontal lines representing 95 per cent c.i. The overall summary effect estimate and 95 per cent c.i. are indicated by the black diamond below. 
Fever and systemic inflammatory response associated with infections may influence stroke recovery [30]. Infections, especially pneumonia usually lead to increased mortality and poor functional outcome $[6,25,26]$. Therefore, there is strong rationale to investigate the effect of antibiotic prophylaxis on patients with acute stroke.

The evidence included in the present meta-analysis showed that preventive antibiotic therapy reduced the occurrence of infection. Several issues could confound the results. Firstly, only one study by Kalra [17] did not show reduced post-stroke pneumonia. One possible reason could be the high-quality care provided at the stroke units participating in the trial. Post-stroke pneumonia is mostly due to aspiration of oropharyngeal secretions promoted by dysphagia, dependent feeding, teeth decay and reduced or ineffective cough. Preventive antibiotic therapy did not add to existing preventive measures including regular suction, positioning, modified diets and early search of infections to start antibiotic treatment [31]. Secondly, definition of infection differed substantially among included studies. One study [20] did not describe the definition of infection in detail. In three studies [16, 17, 19], diagnosis of infection was judged by using criteria of the United States Centers for Disease Control and Prevention [4]. Less strict definitions could overestimate the number of infections, especially in open label studies. Thirdly, to prevent infections, the antibiotic should cover the common causative organisms in post-stroke infections such as pneumonia and urinary tract infections. The most common causative bacteria of pneumonia are taphylococcus aureus and gram-negative bacteria such as Klebsiella pneumoniae, Pseudomonas aeruginosa, Escherichia coli or Enterobacter spp [5]. Escherichia coli and Staphylococcus saprophyticus are commonly identified in patients with urinary tract infection [5]. Antibiotics in six trials [9, 15-17, 19, 20] covered these most common bacteria. However, minocycline in one study [18] inadequately covered the antimicrobial spectrum in patients with acute stroke. The main aim of this study was to investigate a possible neuroprotective effect of minocycline, not to assess the role of this medicine in prevention of the post-stroke infection. Another potential neuroprotective antibiotic used in one study [19] is ceftriaxone, which has a combination of effective coverage of antimicrobial spectrum and neuroprotective properties [19]. Yet, it still failed at the confirmatory trial stage.

The result of this meta-analysis indicated that preventive antibiotic had no effect on overall post-stroke mortality. Several factors might influence the results. As we know, mortality in acute stroke varied between $15 \%$ and $25 \%$ [32]. However, most of included trials [9, 15, 16, $18-20]$ had low mortality $(0 \%-15 \%)$ and only one trial [17] had relatively high mortality (29\%). Three studies exclude patients with a short life expectancy. Inclusion of less severe patients in this meta-analysis might overestimate the efficacy of antibiotic prophylaxis due to the low mortality. Severe patients might benefit the most from preventive antibiotic treatment. Infection rate was associated with the patients' clinical condition. Studies including patients with a higher stroke severity or lower levels of consciousness showed higher infection rates, in particular for pneumonia [5]. Another possibility is that antibiotic can prevent the early or mild infections from

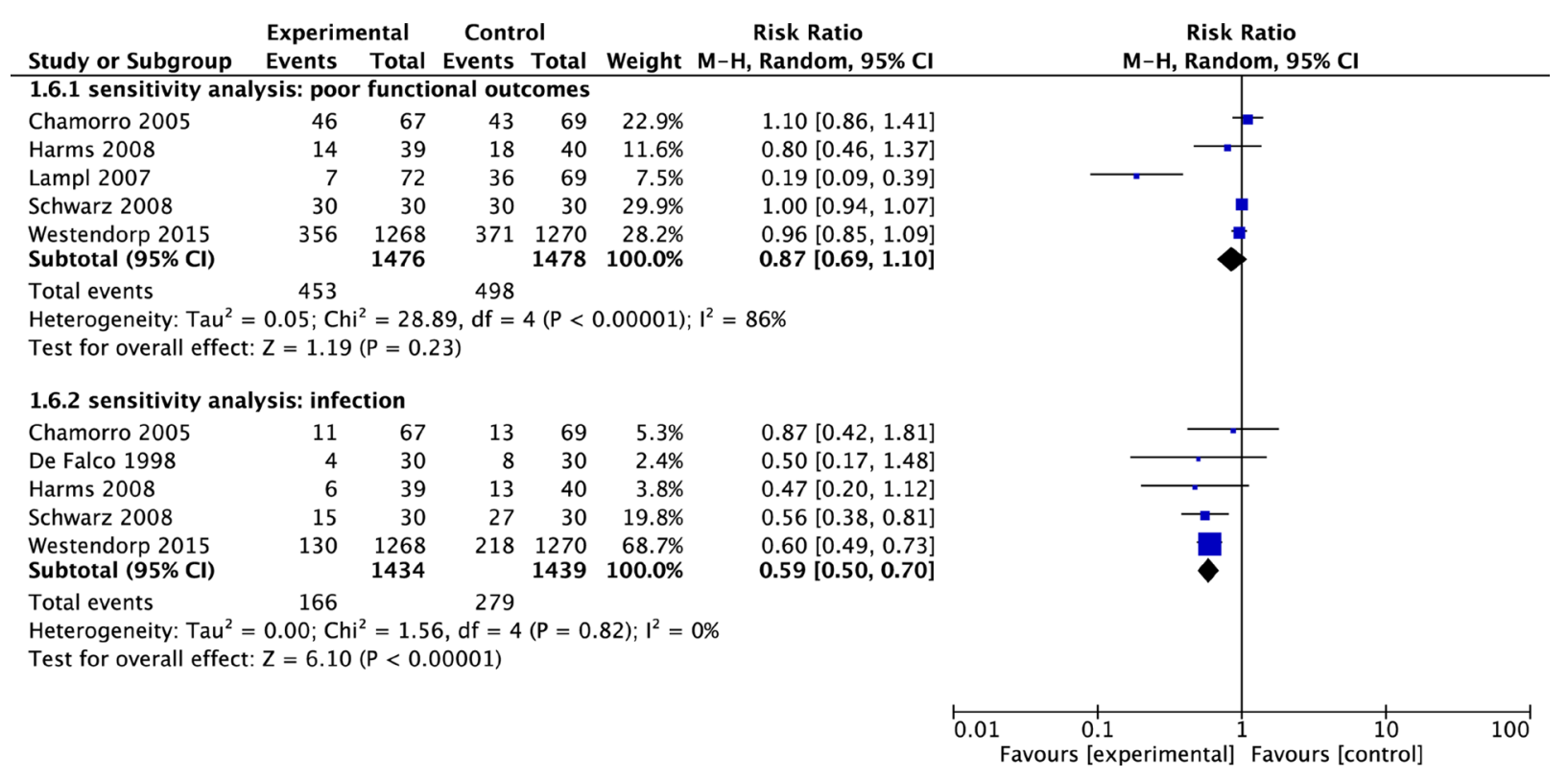

Figure 6: Sensitivity analysis. The summary effect estimate (risk ratio, RR) for individual randomized controlled trials (RCTs) are indicated by green rectangles (the size of the rectangle is proportional to the study weight), with the black horizontal lines representing 95 per cent c.i. The overall summary effect estimate and 95 per cent c.i. are indicated by the black diamond below. 
happening, but for those patients with severe infections, it might also be too severe for antibiotic prophylaxis to reduce the mortality.

Several studies showed that infections were associated with mortality and poor functional outcome $[6,25,26]$. Antibiotic did reduce the post-stroke infection in our meta-analysis, but gave no reason for the fact that decrease of infection rate did not result into a decreased mortality or improve in functional outcome. Poor functional outcome (dependency) was measured with $\mathrm{mRS}$ or BI in activities of daily living. Poor functional outcome is the most important measure of outcome since the aim of therapy should not only be to reduce death but also to reduce disability and dependency in survivors. Infections could affect outcome in several ways. Firstly, they lead to immobilization, general frailty and a delay in rehabilitation due to prolonged hospital stay [5,33]. More importantly, immunological effects of infections could worsen outcome. Evidence from experimental studies suggests that infection promotes antigen presentation and autoimmunity against the brain [34]. Post-stroke infections include pneumonia, UTI, skin and intravenous line infections, sepsis, cholecystitis, etc. A recent study implied that UTI was not associated with functional outcome [26]. Pneumonia is a well-recognized predictor of poor outcome in stroke patients $[6,7,25,26]$. Functional outcome after a stroke is determined largely by the nature of incident stroke, age and premorbid function [26]. Post-stroke pneumonia might be a marker or bystander of stroke severity, and prophylactic antibiotic might not change the course of disease [19]. Infections after stroke most likely result from complex interactions of bacterial, chemical, mechanical (e.g., indwelling catheters), and immunological mechanisms that might not be prevented by antibiotics alone [17] It is therefore possible that toxic effects of treatment as well as the infections themselves mediate the poor outcomes seen in infected stroke patients [35]. To our knowledge, a comprehensive analysis of outcome and infectious complications in stroke patients has not become available in the literature. Moreover, in real-world practice, when diagnosed post-stroke infection in control group patients, early treatment might be just as effective as preventive antibiotic treatment.

Like all medicines, antibiotics may have the potential to cause adverse effects including gastrointestinal problems, allergic reactions, bone marrow suppression, ototoxicity, neurotoxicity and nephrotoxicity. Antibiotic resistance can be another unintended effect of taking antibiotics. Our results suggested that few adverse events occurred in both treatment groups and the incidence of adverse events was low and equal in both groups basically. At least, preventive antibiotic treatment is safe.

Two trials $[15,16]$ used a double-blinded design and four trials $[9,17-19]$ used an open-label design. The open-label trial is a type of clinical trial in which both the researchers and participants know which treatment is being administered. The open-intervention allocation can influence physician diagnosis of post-stroke infections and other outcomes, which could lead to an overestimation of a possible effect. This detection bias might have been minimized by use of blinded assessment of endpoint. Hence, the sub-analysis of trial design indicated that the pooled results were robust.

We have to consider the possibility that antibiotics of different classes may differentially affect stroke outcome independent of infection. For example, both $\beta$-lactam antibiotics and minocycline have neuroprotective properties [36], while fluoroquinolone antibiotics may be neurotoxic. Zierath [37] designed a study which compared behavioral and histological outcomes from stoke in Lewis rats treated with ceftiofur and enrofloxacin. They found no individual antibiotic class was associated with a functional benefit, but rats which were given enrofloxacin had significantly worse functional outcomes. A clinical trial [15] produced the similar result that levofloxacin did not prevent infection and was associated with worse outcome. The neurotoxic mechanisms of Fluoroquinolone is unclear and maybe referred to the inhibition of $\gamma$-aminobutyric acid- A receptor binding, and action at the $\mathrm{N}$-methyl-D-aspartate receptor or alteration of $\mathrm{K}+$ currents [37]. Several studies have showed neuroprotective effects of minocycline and ceftriaxone, yet neither has succeeded at the confirmatory trial stages $[19,38]$. Although no benefit of antibiotics was proved in the treatment of patients with acute stroke, the potential neuroprotective properties confirmed in preclinical studies deserve more investigation. Given the existing evidence that antibiotic administration is safe and well tolerated, the neuroprotective potential of antibiotics should be explored continually in future studies. Furthermore, since the immunological mechanisms and immunodepression take effect immediately and last for a few weeks after the stroke, the time window before start of preventive antibiotic therapy (up to 48 hours) might be too long, and the duration of preventive antibiotic therapy might be too short [39].

Several potential limitations should be appreciated in our analysis. First of all, the conclusion provided in a meta-analysis is only as reliable as the methods used to assess the effect in the primary studies [40]. It also does not overcome problems which inherent in the design of eligible studies. The heterogeneity should be considered, which included the difference in the types and severity of stroke patients, the dose, route and duration of different antibiotics and the diagnostic criteria used for infection. For future studies, standardized definitions of post-stroke infection and time of follow-up are preferable. Secondly, the included studies used insensitive measure methods. Current researchers employed $\mathrm{BI}$ and $\mathrm{mRS}$ as the primary measure of functional recovery. However, sensitivity of $\mathrm{BI}$ and $\mathrm{mRS}[41,42]$ is poor across the range of possible outcomes, particularly in minor or more severe strokes. 
So multidimensional approaches used to evaluate stroke recovery and reliable biomarkers used to objectively evaluate the efficacy of interventions are necessary for future large clinical trials. Thirdly, despite our extensively searching for relevant studies using multiple databases and multiple search items; however, there were difficulties in obtaining unpublished studies. Considering the fact that unpublished trials were mostly those with negative results, unpublished data might just strengthen rather than alter the negative results of this meta-analysis [43]. Fourth, we restricted our search to studies published in English, which potentially led to language bias. Fifth, the findings of present meta-analysis might be impacted by stroke type, stroke severity and patient's age. Unfortunately, we did not perform subgroup analysis according to stroke type, stroke severity and patient's age due to lack of detailed data. Given that only 418 patients with hemorrhagic stroke were included in three studies [15, 17, 19], it may be necessary to further investigate the efficacy of antibiotics prophylaxis in the treatment of acute hemorrhagic stroke. Fifth, publication bias and meta-regression for included studies was not detected because of the limited number (below $<10$ ) of eligible studies, which might influence the results. Sixth, theoretically, studies with a more tailored approach using stricter inclusion criteria (e.g., the early use of antibiotics with adequate duration, more severe patients) and including biomarkers might identify patients who could benefit from preventive antibiotic therapy after stroke. Thus, larger RCTs are required to initiate in the near future. Seventh, "infection" and "acute stroke" had different and heterogeneous definitions in the included different studies and thus readers should to interpret the meta-analysis results with caution.

\section{MATERIALS AND METHODS}

We designed and performed this systematic review and meta-analysis and reported the results according to Cochrane Handbook for systematic review and the Preferred Reporting Items for Systematic Reviews and Meta-analysis (PRISMA) statement [44] respectively. The protocol was prospectively registered at PROSPERO online database and a registration number of CRD42015026980 has been assigned (available at: http:// www.crd.york.ac.uk/prospero/). The informed consent and ethical approval were not required because of this present study was conducted based upon the information from previous studies.

\section{Eligibility criteria}

We designed inclusion criteria based on PICOS principle as following: (1) study design: RCTs investigating the efficacy of antibiotic prophylaxis versus comparisons including placebo or standard treatment; (2) eligible participants: acute stroke patients aged 18 years and older; (3) intervention: antibiotic prophylaxis was administered within 48 hours after stroke onset; and (4) outcomes: reported at least one of outcomes of interest including infection or mortality.

Studies met following criteria were excluded from the present study: studies published in non-English language; the sample size of less than 20 patients in each group; non-original researches such as reviews, expert opinions, letters, commentaries, editorials and research protocol.

\section{Search methods}

We designed two-step search strategy for capturing all potential citations. (1) Three target databases including PubMed, EMBASE and the Cochrane library were electronically searched, and then the reference lists of eligible articles were checked after screened all records identified at initial search period. The initial search was performed on August 2015 and the updated notification was custom daily until September 2015. Following terms were used: 'stroke', 'brain ischemia', 'cerebral ischemia*', 'brain hemorrhage*', 'cerebral stroke*', 'cerebral infarction', 'intracranial hemorrhages', 'cerebrovascular accident', 'antibiotic prophylaxis' and 'preventive antibiotic'. We used Boolean operators (OR, AND, and NOT) constructed all search algorithms. Languages restriction was not imposed in our study. We documented all search algorithms in electronic supplementary material (Supplementary Material of Search Algorithm).

\section{Study selection}

Two independent authors firstly judged the eligibility of the relevant articles through screening titles and abstracts, and checking full-text to determine whether a certain study can be included. Any disagreements on eligibility were resolved by consulting a third reviewer until a consensus was reached.

\section{Data extraction}

Two investigators independently abstracted data from all eligible studies using a predesigned standard data extraction form. Following information including first author's name, year of publication, inclusion and exclusion criteria sample size, intervention and outcomes of interest were abstracted. Discrepancies were resolved in consultation with a third reviewer.

\section{Assessment of risk of bias}

The Cochrane Risk of Bias tool was used to appraise the risk of bias of all included studies [45]. The following domains were evaluated accordingly: random sequence generation, allocation concealment, blinding (participants, personnel and outcome assessment), incomplete outcome 
data, selective reporting and other sources of bias [45]. Based upon the matching level between abstracted information and judgment criteria, each study might be rated to be as low, unclear or high risk of bias [45]. A third author will be consulted if any divergence was identified.

\section{Outcomes measures}

\section{Primary outcomes}

(1) mortality at the end of follow-up and (2) infection rate within the first 2 weeks after stroke onset.

\section{Secondary outcomes}

(1) poor functional outcome (dependency): defined as a Barthel Index $(\mathrm{BI})<60$ or a score on modified Rankin score (mRS) $>2$ at the end of follow-up; and (2) medication-related adverse events (AEs).

\section{Statistical analysis}

We used Review Manager (RevMan) version 5.3.5 (Cochrane Collaboration, Copenhagen, Denmark) to perform all meta-analyses. We estimated the risk ratio (RR) with $95 \%$ confidence interval (CI) to express dichotomous outcomes including mortality, infection and poor functional outcome. The DerSimonian-Laird randomeffect model accounting for different sources of variation from studies was used as a conservative approach to calculate effect estimates. Heterogeneity across studies was examined with inconsistency analysis $\left(I^{2}\right)$ and $Q$-test (with a $P$-value $<0.10$ considered substantial) [46]. A $I^{2}<50 \%$ shows homogeneous, a $I^{2} \geq 50 \%$ indicates the existence of heterogeneity. We performed subgroup analyses according to study design (double-blinded and open-label). Moreover, we carried out sensitivity analyses based on study design. All reported $P$ values were 2-tailed and a 0.05 was seen to be significance. The publication bias and meta-regression will not be evaluated when the number of eligible studies was less than 10 .

\section{CONCLUSIONS}

In adults with acute stroke, antibiotic prophylaxis did reduce the occurrence of infection, but did not reduce mortality and poor functional outcome. Sensitivity analysis showed that infection rate and poor functional outcome were stable. Thus, antibiotic prophylaxis may not be recommended for acute stroke patients. No major side-effects of preventive antibiotic therapy were reported.

\section{Abbreviations}

RCTs $=$ randomized controlled trials, PRISMA $=$ Preferred Reporting Items for Systematic Reviews and Meta-analysis, $\mathrm{MeSH}=$ medical subject heading, BI
$=$ Barthel Index, $\mathrm{mRS}=$ modified Rankin score, $\mathrm{AEs}=$ adverse events, RevMan $=$ Review Manager, $R R=$ risk ratio, $\mathrm{CI}=$ confidence interval, $\mathrm{I}^{2}=$ inconsistency analysis, NIHSS = National Institute of Health Stroke Scale, CNS = Canadian Neurological Scale, ID = identity card, MRSA $=$ methicillin-resistant Staphylococcus aureus, $\mathrm{CDT}=$ Clostridium difficile toxin, UTI = urinary tract infection .

\section{Authors' contributions}

Y.-G.X., X.T., W.-Q.C., S.Z. and S.Z. conceived and designed the protocol. Y.-G.X., X.T., S.Z. and W.-D.R. selected studies, collected and analyzed the data. Y.-G.X., X.T. and S.Z. and performed statistical analysis. W.-D.R., Z.-M.Y., and G.-T.Y. assessed the quality of included studies and evidence. Y.-G.X., X.T., W.-Q.C., S.Z. and S.Z. drafted the manuscript. Y.-G.X., X.T., W.-Q.C. and G.-T.Y. critically edited the language. All authors read and approved the final manuscript.

\section{ACKNOWLEDGMENTS}

We would like to appreciate the helps proposed by associated editors and anonymous referees.

\section{CONFLICTS OF INTEREST}

All authors reviewed the whole manuscript and approved to submit this manuscript to be considered for publication in Oncotarget. No conflicts interests are existed.

\section{FUNDING}

This article was not supported by any financial sources.

\section{REFERENCES}

1. Mozaffarian D, Benjamin EJ, Go AS, Arnett DK, Blaha MJ, Cushman M, de Ferranti S, Despres JP, Fullerton HJ, Howard VJ, Huffman MD, Judd SE, Kissela BM, et al. Heart disease and stroke statistics - 2015 update: a report from the American Heart Association. Circulation. 2015; 131:e29-322.

2. Naghavi M, Wang $H$, Lozano R, Davis A, Liang $\mathrm{X}$, Zhou M, Vollset SE, Ozgoren AA, Abdalla S, Abd-Allah F, Abdel Aziz MI, Abera SF. Global, regional, and national age-sex specific all-cause and cause-specific mortality for 240 causes of death, 1990-2013: a systematic analysis for the Global Burden of Disease Study 2013. Lancet. 2015; 385:117-171.

3. Kumar S, Selim MH, Caplan LR. Medical complications after stroke. Lancet Neurol. 2010; 9:105-118.

4. Horan TC, Andrus M, Dudeck MA. CDC/NHSN surveillance definition of health care-associated infection 
and criteria for specific types of infections in the acute care setting. Am J Infect Control. 2008; 36:309-332.

5. Westendorp WF, Nederkoorn PJ, Vermeij JD, Dijkgraaf MG, van de Beek D. Post-stroke infection: a systematic review and meta-analysis. BMC Neurol. 2011; 11:110.

6. Finlayson O, Kapral M, Hall R, Asllani E, Selchen D, Saposnik G. Risk factors, inpatient care, and outcomes of pneumonia after ischemic stroke. Neurology. 2011; 77:1338-1345.

7. Koennecke HC, Belz W, Berfelde D, Endres M, Fitzek S, Hamilton F, Kreitsch P, Mackert BM, Nabavi DG, Nolte CH, Pohls W, Schmehl I, Schmitz B, et al. Factors influencing in-hospital mortality and morbidity in patients treated on a stroke unit. Neurology. 2011; 77:965-972.

8. Westendorp WF, Vermeij JD, Vermeij F, Den Hertog HM, Dippel DW, van de Beek D, Nederkoorn PJ. Antibiotic therapy for preventing infections in patients with acute stroke. Cochrane Database Syst Rev. 2012; 1:Cd008530.

9. Schwarz S, Al-Shajlawi F, Sick C, Meairs S, Hennerici MG. Effects of prophylactic antibiotic therapy with mezlocillin plus sulbactam on the incidence and height of fever after severe acute ischemic stroke: the Mannheim infection in stroke study (MISS). Stroke. 2008; 39:1220-1227.

10. Hetze S, Engel O, Romer C, Mueller S, Dirnagl U, Meisel C, Meisel A. Superiority of preventive antibiotic treatment compared with standard treatment of poststroke pneumonia in experimental stroke: a bed to bench approach. J Cereb Blood Flow Metab. 2013; 33:846-854.

11. Meisel C, Prass K, Braun J, Victorov I, Wolf T, Megow D, Halle E, Volk HD, Dirnagl U, Meisel A. Preventive antibacterial treatment improves the general medical and neurological outcome in a mouse model of stroke. Stroke. 2004; 35:2-6.

12. Dalhoff A, Shalit I. Immunomodulatory effects of quinolones. Lancet Infect Dis. 2003; 3:359-371.

13. Rothstein JD, Patel S, Regan MR, Haenggeli C, Huang YH, Bergles DE, Jin L, Dykes Hoberg M, Vidensky S, Chung DS, Toan SV, Bruijn LI, Su ZZ, et al. Beta-lactam antibiotics offer neuroprotection by increasing glutamate transporter expression. Nature. 2005; 433:73-77.

14. Thone-Reineke C, Neumann C, Namsolleck P, Schmerbach K, Krikov M, Schefe JH, Lucht K, Hortnagl H, Godes M, Muller S, Rumschussel K, Funke-Kaiser H, Villringer A, et al. The beta-lactam antibiotic, ceftriaxone, dramatically improves survival, increases glutamate uptake and induces neurotrophins in stroke. J Hypertens. 2008; 26:2426-2435.

15. Chamorro A, Horcajada JP, Obach V, Vargas M, Revilla M, Torres F, Cervera A, Planas AM, Mensa J. The Early Systemic Prophylaxis of Infection After Stroke study: a randomized clinical trial. Stroke. 2005; 36:1495-1500.

16. Harms H, Prass K, Meisel C, Klehmet J, Rogge W, Drenckhahn C, Gohler J, Bereswill S, Gobel U, Wernecke KD, Wolf T, Arnold G, Halle E, et al. Preventive antibacterial therapy in acute ischemic stroke: a randomized controlled trial. PLoS One. 2008; 3:e2158.

17. Kalra L, Irshad S, Hodsoll J, Simpson M, Gulliford M, Smithard D, Patel A, Rebollo-Mesa I. Prophylactic antibiotics after acute stroke for reducing pneumonia in patients with dysphagia (STROKE-INF): a prospective, cluster-randomised, open-label, masked endpoint, controlled clinical trial. Lancet. 2015; 386:1835-44.

18. Lampl Y, Boaz M, Gilad R, Lorberboym M, Dabby R, Rapoport A, Anca-Hershkowitz M, Sadeh M. Minocycline treatment in acute stroke: an open-label, evaluator-blinded study. Neurology. 2007; 69:1404-1410.

19. Westendorp WF, Vermeij JD, Zock E, Hooijenga IJ, Kruyt ND, Bosboom HJ, Kwa VI, Weisfelt M, Remmers MJ, ten Houten R, Schreuder AH, Vermeer SE, van Dijk EJ, et al. The Preventive Antibiotics in Stroke Study (PASS): a pragmatic randomised open-label masked endpoint clinical trial. Lancet. 2015; 385:1519-1526.

20. De Falco FA, Santangelo R, Majello L, Angelone P. Antimicrobial prophylaxis in the management of ischemic stroke. Riv Neurobiol. 1998; 44:63-67.

21. Adams HP Jr, del Zoppo G, Alberts MJ, Bhatt DL, Brass L, Furlan A, Grubb RL, Higashida RT, Jauch EC, Kidwell C, Lyden PD, Morgenstern LB, Qureshi AI, et al. Guidelines for the early management of adults with ischemic stroke: a guideline from the American Heart Association/American Stroke Association Stroke Council, Clinical Cardiology Council, Cardiovascular Radiology and Intervention Council, and the Atherosclerotic Peripheral Vascular Disease and Quality of Care Outcomes in Research Interdisciplinary Working Groups: The American Academy of Neurology affirms the value of this guideline as an educational tool for neurologists. Circulation. 2007; 115:e478-534.

22. Tong X, Kuklina EV, Gillespie C, George MG. Medical complications among hospitalizations for ischemic stroke in the United States from 1998 to 2007. Stroke. 2010; 41:980-986.

23. Van De Beek D, Wijdicks EFM, Vermeij FH, De Haan RJ, Prins JM, Spanjaard L, Dippel DWJ, Nederkoorn PJ. Preventive antibiotics for infections in acute stroke: A systematic review and meta-analysis. Arch Neurol. 2009; 66:1076-1081.

24. Smith CJ, Kishore AK, Vail A, Chamorro A, Garau J, Hopkins SJ, Di Napoli M, Kalra L, Langhorne P, Montaner J, Roffe C, Rudd AG, Tyrrell PJ, et al. Diagnosis of StrokeAssociated Pneumonia: Recommendations From the Pneumonia in Stroke Consensus Group. Stroke. 2015; 46:2335-2340.

25. Popovic N, Stefanovic-Budimkic M, Mitrovic N, Urosevic A, Milosevic B, Pelemis M, Jevtovic D, BeslacBumbasirevic L, Jovanovic D. The frequency of poststroke infections and their impact on early stroke outcome. J Stroke Cerebrovasc Dis. 2013; 22:424-429.

26. Rohweder G, Ellekjaer H, Salvesen O, Naalsund E, Indredavik B. Functional outcome after common poststroke complications occurring in the first 90 days. Stroke. 2015; 46:65-70. 
27. Fu Y, Liu Q, Anrather J, Shi FD. Immune interventions in stroke. Nature reviews Neurology. 2015; 11:524-535.

28. Schwarz S. Prophylactic Antibiotic Therapy for Preventing Poststroke Infection. Neurotherapeutics. 2016; 13:783-790.

29. Famakin BM. The Immune Response to Acute Focal Cerebral Ischemia and Associated Post-stroke Immunodepression: A Focused Review. Aging Dis. 2014; 5:307-326.

30. Poisson SN, Johnston SC, Josephson SA. Urinary tract infections complicating stroke: mechanisms, consequences, and possible solutions. Stroke. 2010; 41:e180-184.

31. Titsworth WL, Abram J, Fullerton A, Hester J, Guin P, Waters MF, Mocco J. Prospective quality initiative to maximize dysphagia screening reduces hospital-acquired pneumonia prevalence in patients with stroke. Stroke. 2013; 44:3154-3160.

32. van der Worp HB, van Gijn J. Clinical practice. Acute ischemic stroke. N Engl J Med. 2007; 357:572-579.

33. Vermeij FH, Scholte op Reimer WJ, de Man P, van Oostenbrugge RJ, Franke CL, de Jong G, de Kort PL, Dippel DW. Stroke-associated infection is an independent risk factor for poor outcome after acute ischemic stroke: data from the Netherlands Stroke Survey. Cerebrovasc Dis. 2009; 27:465-471.

34. Smith CJ, Lawrence CB, Rodriguez-Grande B, Kovacs KJ, Pradillo JM, Denes A. The immune system in stroke: clinical challenges and their translation to experimental research. Journal of neuroimmune pharmacology. 2013; 8:867-887.

35. Miller EC, Elkind MS. Infection and Stroke: an Update on Recent Progress. Curr Neurol Neurosci Rep. 2016; 16:2.

36. Stock ML, Fiedler KJ, Acharya S, Lange JK, Mlynarczyk GS, Anderson SJ, McCormack GR, Kanuri SH, Kondru NC, Brewer MT, Carlson SA. Antibiotics acting as neuroprotectants via mechanisms independent of their antiinfective activities. Neuropharmacology. 2013; 73:174-182.
37. Zierath D, Kunze A, Fecteau L, Becker K. Effect of Antibiotic Class on Stroke Outcome. Stroke. 2015; 46:2287-2292.

38. Kohler E, Prentice DA, Bates TR, Hankey GJ, Claxton A, van Heerden J, Blacker D. Intravenous minocycline in acute stroke: a randomized, controlled pilot study and metaanalysis. Stroke. 2013; 44:2493-2499.

39. Silvestri L, van Saene HK, Milanese M, Ros S, Zandstra DF. Parenteral antibiotics are not enough to prevent pneumonia in stroke. Lancet. 2015; 386:653.

40. Garg AX, Hackam D, Tonelli M. Systematic review and meta-analysis: when one study is just not enough. Clin J Am Soc Nephrol. 2008; 3:253-260.

41. Dromerick AW, Edwards DF, Diringer MN. Sensitivity to changes in disability after stroke: a comparison of four scales useful in clinical trials. J Rehabil Res Dev. 2003; 40:1-8.

42. Quinn TJ, Langhorne P, Stott DJ. Barthel index for stroke trials: development, properties, and application. Stroke. 2011; 42:1146-1151.

43. Easterbrook PJ, Berlin JA, Gopalan R, Matthews DR. Publication bias in clinical research. Lancet. 1991; 337:867-872.

44. Moher D, Liberati A, Tetzlaff J, Altman DG. Preferred reporting items for systematic reviews and meta-analyses: the PRISMA statement. PLoS Med. 2009; 6:e1000097.

45. Julian PT, Higgins SG. (2011.Available from www. cochrane-handbook.org.). Cochrane Handbook for Systematic Review of Interventions Version 5.1.0 [updated March 2011]. The Cochrane Collaboration.

46. Higgins JP, Thompson SG, Deeks JJ, Altman DG. Measuring inconsistency in meta-analyses. BMJ. 2003; 327:557-560. 